\title{
Flexible Photodetectors with High Responsivity and Broad Spectral Response employing Ternary $\mathrm{Sn}_{x} \mathrm{Cd}_{1-x} \mathrm{~S}$ Micro- nanostructures
}

Muhammad Zubair Nawaz ${ }^{1}$, Liu Xu ${ }^{1}$, Xing Zhou ${ }^{1}$, Khizar Hussain Shah ${ }^{1}$, Muhammad Yaqub ${ }^{2}$, Jiale Wang ${ }^{1}$, Binhe $\mathrm{Wu}^{1}$, Chunrui Wang ${ }^{1 *}$

${ }^{1}$ College of Science and Shanghai Institute of Intelligent Electronics and Systems, Donghua University, 2999 Renmin Rd North, Songjiang District, Shanghai 201620, P. R. China

${ }^{2}$ Faculty of Information Technology, Beijing University of Technology, Beijing 100000, China

E-mail: crwang@dhu.edu.cn, zubair@mail.dhu.edu.cn

Characterizations of $\mathrm{Sn}_{\mathrm{x}} \mathrm{Cd}_{1-\mathrm{x}} \mathrm{S}$ nanobelts

Powder X-ray diffraction (XRD) of the as-synthesized $\mathrm{Cd}_{x} \mathrm{Sn}_{1-\mathrm{x}} \mathrm{S}$ and pristine $\mathrm{CdS}$ nanobelts was conducted by a D/max 2550 VB+/PC X-ray diffractometer (Rigaku, Japan) equipped with a rotating anode and a Cu K $\alpha$ radiation source $(\lambda=1.5404 \AA$ ). Raman spectra were recorded at room temperature on an inVia-Reflex micro-Raman spectroscopy system from Renishaw with $532 \mathrm{~nm}$ laser. X-ray photoelectron spectroscopy (XPS) data were obtained using a Escalab 250Xi (Thermo Scientific) spectrometer with an excitation source of Al-Ka radiation. Morphology of $\mathrm{Cd}_{x} \mathrm{Sn}_{1-x} \mathrm{~S}$ nanobelts were determined by a field emission scanning electron microscopy (FESEM) Hitachi S-4800 and field emission transmission electron microscopy (FE-TEM JEM 2100 F) equipped with energy dispersive spectrometer (EDS).

\section{PHOTODETECTOR MEASUREMENT}

The current-voltage (I-V) characteristics of the $\mathrm{Cd}_{x} \mathrm{Sn}_{1-\mathrm{x}} \mathrm{S}$ nanobelt photodetectors were measured by Keithley4200 semiconductor characterization system (SCS) on a probe station at room temperature. Monochromatic light with wavelength $300 \mathrm{~nm}$ to $700 \mathrm{~nm}$ was achieved from a $300 \mathrm{~W}$ xenon lamp with monochromators. 


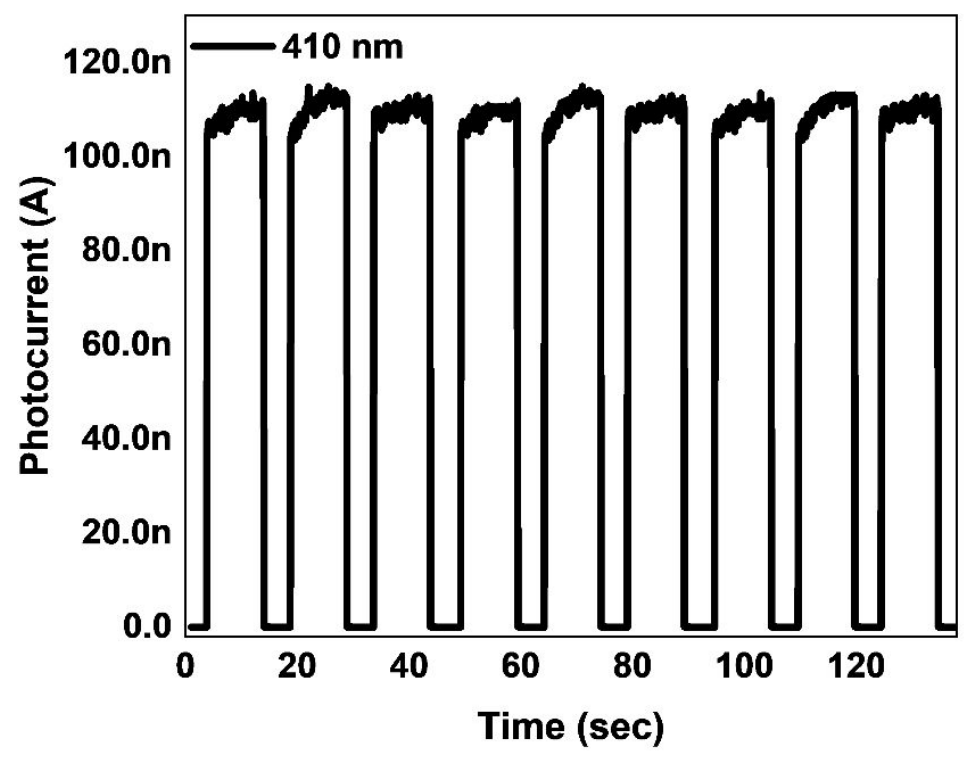

Figure S-1: Photoswitching photocurrent cycles of $\mathrm{Sn}_{x} \mathrm{Cd}_{1-x} \mathrm{~S}$ nanobelt based PD on rigid Si substrate under 410 $\mathrm{nm}$ illumination.

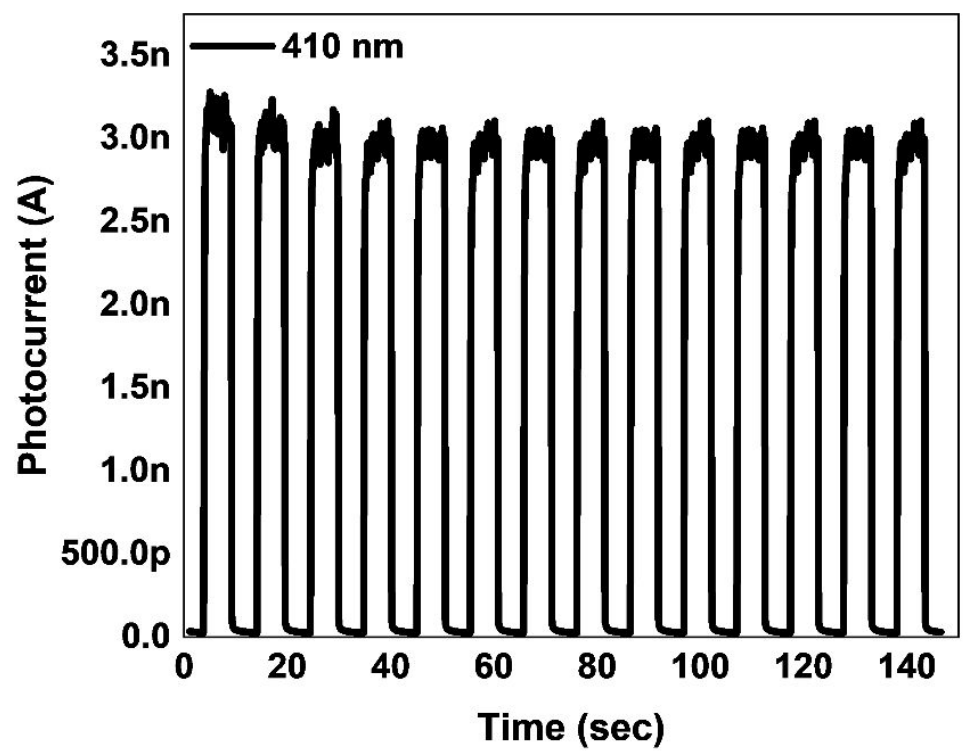

Figure S-2: Photoswitching photocurrent cycles of $\mathrm{Sn}_{x} \mathrm{Cd}_{1-x} \mathrm{~S}$ nanobelt based FPD under $410 \mathrm{~nm}$ illumination. 


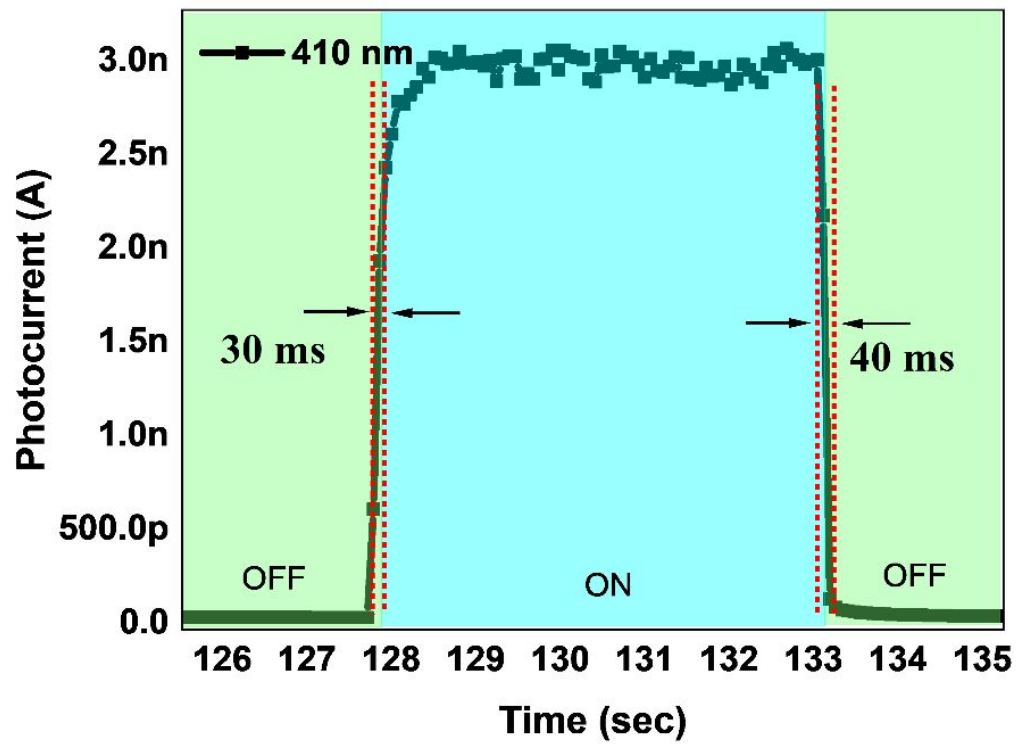

Figure S-3: A selected single cycle of photoswitching photocurrent obtained from Figure 10c. 